\title{
A remark on Xia's theorem concerning quasi-invariant measures
}

\author{
By Yasuji TAKahashi \\ (Received June 22, 1981; Revised November 2, 1981)
}

\section{$\S 1$. Introduction}

Let $E$ and $F$ be Banach spaces, and suppose that $F$ itself is a linear subspace of $E$. Also suppose that the inclusion mapping $J$ of $F$ into $E$ is continuous. Then the author [4, Proposition 3.1] proved the following:

Proposition 1.1. The following implication $(1) \Rightarrow(2)$ holds.

(1) There exists a finite Borel measure on E which is quasi-invariant with respect to $F$.

(2) The adjoint mapping $J^{*}$ of $E^{*}$ into $F^{*}$ is absolutely summing.

In Proposition 1.1, if $E$ is a Hilbert space and $F$ is a Banach space, then the converse implication $(2) \Rightarrow(1)$ also holds (cf. [4, Theorem $A]$ ).

Now we shall consider the following problem.

Problem. Can we show the equivalence of statements (1) and (2) of Proposition 1.1 when $E$ and $F$ belong to some suitable class of Banach spaces?

Concerning this, Xia [7, p. 319, Example 5.3.1] and the author [4, Proposition 4.1.1] proved the following:

THEOREM 1.1. Let $1 \leqq p<\infty, 1 \leqq q \leqq 2$. If we assume that $l^{q} \subset l^{p}\left(a_{n}\right)$, then the following statements are equivalent.

(1) There exists a finite Borel measure on $l^{p}\left(a_{n}\right)$ which is quasiinvariant with respect to $l^{q}$.

(2) The adjoint mapping $J^{*}$ of $\left(l^{p}\left(a_{n}\right)\right)^{*}$ into $\left(l^{q}\right)^{*}$ is absolutely summing, where $J$ denotes the inclusion mapping of $l^{q}$ into $l^{p}\left(a_{n}\right)$.

(3) $\sum a_{n}<\infty$.

The purpose of this note is to give a generalization of the above theorem to a function space, which is stated as follows:

Let $(\Omega, \Sigma, \mu)$ and $(\Omega, \Sigma, \nu)$ be $\sigma$-finite measure spaces. For $1 \leqq p<\infty$, $1 \leqq q<\infty$, we denote by $L^{p}(\mu)$ the Banach space of equivalence classes of real valued measurable functions on $(\Omega, \Sigma, \mu)$ whose $p$ 'th power is integrable, 
and also denote by $L^{q}(\nu)$ the Banach space of equivalence classes of real valued measurable functions on $(\Omega, \Sigma, \nu)$ whose $q$ 'th power is integrable.

Theorem 1.2. Let $1 \leqq p<\infty, 1 \leqq q \leqq 2$. Suppose that two measures $\mu$ and $\nu$ are mutually equivalent, and $L^{q}(\nu) \subset L^{p}(\mu)$. Then the following statements are equivalent.

(1) There exists a finite Borel measure on $L^{p}(\mu)$ which is quasiinvariant with respect to $L^{q}(\nu)$.

(2) The adjoint mapping $J *$ of $\left(L^{p}(\mu)\right) *$ into $\left(L^{q}(\nu)\right) *$ is absolutely summing, where $J$ denotes the inclusion mapping of $L^{q}(\nu)$ into $L^{p}(\mu)$.

REMARK 1.1. In the above theorem, if we assume that the measure $\mu$ is absolutely continuous with respect to the measure $\nu$ (not necessarily equivalent), then the similar result can be proved (cf. Theorem 3.1 of Section 3).

Throughout this note, we assume that all linear spaces are with real coefficients and all measures are nontrivial.

\section{$\S 2 . \quad$ Definitions and lemmas}

Let $E$ be a linear topological space, and let $P$ be a Borel measure on $E$. For an element $x$ in $E$, let $P_{x}$ denote the Borel measure on $E$ defined by

$$
P_{x}(A)=P(A-x) \quad \text { for any Borel set } A \text { of } E \text {. }
$$

Definition 2.1. An element $x$ of $E$ is called an admissible shift for the measure $P$ if $P_{x}$ is absolutely continuous with respect to $P$. The set of admissible shifts of the measure $P$ will be denoted by $M_{P}$.

An element $x$ of $E$ is called a partially admissible shift for the measure $P$ if $P_{x}$ contains a non-trivial component absolutely continuous with respect to $P$. The set of partially adimissible shifts of the measure $P$ will be denoted by $\widetilde{M}_{P}$.

It is easily seen that $M_{P} \subset \widetilde{M}_{P}$, but in general $M_{P}$ does not coincide with $\widetilde{M}_{P .} \quad$ (For details; see [3, Chapter 4, § 19], [6].)

Let $F$ be a linear subspace of $E$. We shall say that the measure $P$ is quasi-invariant with respect to $F$ if $F \subset M_{P}$.

Definition 2.2. Let $1 \leqq p<\infty$. A continuous linear mapping $T$ of a Banach space $E$ into a Banach space $F$ is called $p$-absolutely summing if it takes each weakly $p$-summable sequence $\left\{x_{n}\right\}$ of $E$ into an absolutely $p$ summable sequence $\left\{T\left(x_{n}\right)\right\}$ of $F$.

We shall say "absolutely summing" instead of "1-absolutely summing". For $1 \leqq p \leqq q<\infty$, it is well known (cf. [2, Satz 5]) that if $T$ is $p$ - 
absolutely summing, then $T$ is $q$-absolutely summing.

The following result is a generalization of Proposition 1.1.

LemMA 2.1. (cf. [6, Theorem 4.2])

Let $T$ be a continuous linear mapping of a Banach space $F$ into a Banach space $E$. Then the following implication $(1) \Rightarrow(2)$ holds.

(1) There exists a finite Borel measure $P$ on $E$ such that $T(F) \subset \widetilde{M}_{P}$.

(2) The adjoint mapping $T^{*}$ of $E^{*}$ into $F^{*}$ is absolutely summing.

Now we introduce a sequence space $l^{p}\left(a_{n}\right)$.

Let $1 \leqq p<\infty$, let $\left\{a_{n}\right\}$ be a sequence of positive numbers, and let $l^{p}\left(a_{n}\right)$ denote the totality of real number sequences $\xi=\left\{\xi_{n}\right\}$ which satisfy the condition

$$
\|\boldsymbol{\xi}\|=\left(\sum a_{n}\left|\xi_{n}\right|^{p}\right)^{\frac{1}{p}}<\infty .
$$

$l^{p}\left(a_{n}\right)$ forms a Banach space with respect to the coordinatewise linear operations and the norm (2.1).

Lemma 2.2. (cf. [4, Proposition 4.1.1])

Let $1 \leqq p<\infty, 1 \leqq q \leqq 2$. Let $\left\{a_{n}\right\}$ and $\left\{b_{n}\right\}$ be two sequences of positive numbers, respectively. Suppose that $l^{q}\left(b_{n}\right) \subset l^{p}\left(a_{n}\right)$. Then the following two statements are equivalent.

(1) There exists a finite Borel measure on $l^{p}\left(a_{n}\right)$ which is quasi-invariant with respect to $l^{q}\left(b_{n}\right)$.

(2) $\sum a_{n} / b_{n}^{\frac{p}{q}}<\infty$.

\section{$\S 3$. Main theorem and other results}

Let $(\Omega, \Sigma, \mu)$ and $(\Omega, \Sigma, \nu)$ be $\sigma$-finite measure spaces. The $\mu$-measurable set $A$ of positive measure is called a $\mu$-atom if for any $\mu$-measurable subset $B$ of $A$, we have either $\mu(B)=0$ or $\mu\left(A \cap B^{c}\right)=0$. Denote by $L^{0}(\mu)$ the linear space of equivalence classes of real valued measurable functions on $(\Omega, \Sigma, \mu)$, and denore by $L^{0}(\nu)$ the linear space of equivalence classes of real valued measurable functions on $(\Omega, \Sigma, \nu)$. Suppose that the measure $\mu$ is absolutely continuous with respect to the measure $\nu$. Then, for two measurable functions $f(\omega)$ and $g(\omega)$ on $(\Omega, \Sigma)$, " $f(\omega)=g(\omega)$ for $\nu$-a. e. $\omega$ " implies that " $f(\omega)=g(\omega)$ for $\mu$-a.e. $\omega$ ". Thus the natural mapping $J$ of $L^{0}(\nu)$ into $L^{0}(\mu)$ can be defined.

Let $1 \leqq p<\infty, 1 \leqq q<\infty$. Since the Banach $L^{q}(\nu)$ is a linear subspace of $L^{0}(\nu), J$ is a linear mapping of $L^{q}(\nu)$ into $L^{0}(\mu)$. Also since $L^{0}(\mu)$ is a 
complete linear metric space with respect to the topology of measure convergence, it follows from the closed graph theorem that $J$ is a continuous linear mapping of $L^{q}(\nu)$ into $L^{0}(\mu)$. Thus, $J\left(L^{q}(\nu)\right)$ becomes a Banach space with respect to the quotient norm. We remark that $J\left(L^{q}(\nu)\right) \cap L^{p}(\mu)$ also becomes a Banach space with respect to the following norm $\|\cdot\|$;

$$
\|f\|=\|f\|_{p}+\|f\|_{q} \quad \text { for any } f \in J\left(L^{q}(\nu)\right) \cap L^{p}(\mu)
$$

where $\|\cdot\|_{p}$ and $\|\cdot\|_{q}$ denote the norms in Banach spaces $L^{p}(\mu)$ and $J\left(L^{q}(\nu)\right)$, respectively.

For simplicity, we shall write $F$ instead of $J\left(L^{q}(\nu)\right) \cap L^{p}(\mu)$ in the ensuing discussions. Denote by $T$ the inclusion mapping of a Banach space $F$ into a Banach space $L^{p}(\mu)$.

Then our main theorem is stated as follows :

THEOREM 3.1. Let $1 \leqq p<\infty, 1 \leqq q \leqq 2$. Then the following five statements are equivalent.

(1) There exists a finite Borel measure on $L^{p}(\mu)$ which is quasiinvariant with respect to $F$.

(2) There exists a finite Borel measure $P$ on $L^{p}(\mu)$ such that $F \subset \widetilde{M}_{P}$.

(3) The adjoint mapping $T^{*}$ of $\left(L^{p}(\mu)\right)^{*}$ into $F^{*}$ is absolutely summing. ming.

(4) The adjoint mapping $T^{*}$ of $\left(L^{p}(\mu)\right)^{*}$ into $F^{*}$ is p-absolutely sum-

(5) For any $X_{n} \subset \Omega$ which is measurable and mutually disjoint with $0<\mu\left(X_{n}\right)<\infty, 0<\nu\left(X_{n}\right)<\infty$, the following inequality

$$
\sum \mu\left(X_{n}\right) / \nu\left(X_{n}\right)^{\frac{p}{q}}<\infty
$$

holds.

To prove this theorem, the following lemma is very useful.

Lemma 3.1. Under the hypotheses of Theorem 3.1, the following implication $(1) \Rightarrow(2)$ holds.

(1) The adjoint mapping $T^{*}$ of $\left(L^{p}(\mu)\right)^{*}$ into $F^{*}$ is p-absolutely summing.

(2) For any $X_{n} \subset \Omega$ which is measurable and mutually disjoint with $0<\mu\left(X_{n}\right)<\infty, 0<\nu\left(X_{n}\right)<\infty$, the following inequality

$$
\sum \mu\left(X_{n}\right) /\left(\mu\left(X_{n}\right)^{\frac{1}{p}}+\nu\left(X_{n}\right)^{\frac{1}{q}}\right)^{p}<\infty
$$

holds.

This proof can be done by a quite similar way as in the proof of Pro- 
position 4.2.1 of [4], and so we omit it.

Now we shall prove our main theorem.

Proof of Theorem 3.1.

$(1) \Rightarrow(2)$ : This is obvious.

$(2) \Rightarrow(3)$ : This follows from Lemma 2.1.

$(3) \Rightarrow(4)$ : This follows from Satz 5 of [2].

$(4) \Rightarrow(5)$ : Let $\left\{X_{n}\right\}$ be a sequence of mutually disjoint measurable subsets of $\Omega$ such that $0<\mu\left(X_{n}\right)<\infty, 0<\nu\left(X_{n}\right)<\infty$. Suppose that the statement (4) holds. Then it follows from Lemma 3.1 that the inequality (3.3) holds, so that for a given $\varepsilon$ with $0<\varepsilon<1$, there exists a natural number $N$ such that

$$
\mu\left(X_{n}\right) /\left(\mu\left(X_{n}\right)^{\frac{1}{p}}+\nu\left(X_{n}\right)^{\frac{1}{q}}\right)^{p}<\varepsilon^{p} \quad \text { for all } n \geqq N .
$$

Hence we have

$$
\mu\left(X_{n}\right)^{\frac{1}{p}}<\frac{\varepsilon}{1-\varepsilon} \nu\left(X_{n}\right)^{\frac{1}{q}} \quad \text { for all } n \geqq N .
$$

Putting $\varepsilon=\frac{1}{2}$; from (3.3) and (3.5), we have

$$
\sum \mu\left(X_{n}\right) /\left(\nu\left(X_{n}\right)^{\frac{1}{q}}+\nu\left(X_{n}\right)^{\frac{1}{q}}\right)^{p}<\infty .
$$

This shows that the inequality (3.2) certainly holds.

$(5) \Rightarrow(1)$ : Since $(\Omega, \Sigma, \nu)$ is a $\sigma$-finite measure space, it can be shown that there exist mutually disjoint measurable subsets $X$ and $Y$ such that $\Omega=X \cup Y$, where neither $X$ nor any of its measurable subsets is an atom, and $Y$ is a union of an at most countable number of atoms $Y_{k}$ with $0<$ $\nu\left(Y_{k}\right)<\infty(k=1,2, \cdots \cdots)$. Let $\left\{Y_{k_{n}}\right\}$ be a subsequence of $\left\{Y_{k}\right\}$ such that $\mu\left(Y_{K_{n}}\right)>0(n=1,2, \cdots \cdots)$.

For simplicity, we shall write $Y_{n}$ instead of $Y_{k_{n}}$. Then it is easy to show that $Y_{n}$ is a $\mu$-atom and $\mu\left(Y_{n}\right)<\infty$. Let $f(\omega)$ denote the RadonNikodym derivative of $\mu$ with respect to $\nu$. Then we have

$$
\mu(A)=\int_{A} f(\omega) d \nu(\omega) \quad \text { for any } A \text { of } \Sigma .
$$

Now suppose that the statement (5) holds. Then we can show that $\mu(X)=0$. For if $\mu(X)>0$, then from (3.6), there exist a positive constant $C$ and measurable subset $Z$ of $X$ such that $\nu(Z)>0$ where $Z=\{\omega \in X$; $f(\omega) \geqq C\}$. Since neither $Z$ nor any of its measurable subsets is a $\nu$-atom, there exists a sequence of mutually disjoint measurable subsets $Z_{n}$ of $Z$ such that 


$$
\sum \nu\left(Z_{n}\right)^{1-\frac{p}{q}}=\infty
$$

Also since

$$
\mu\left(Z_{n}\right)=\int_{Z_{n}} f(\omega) d \nu(\omega) \geqq C \nu\left(Z_{n}\right),
$$

hence it follows from (3.7) that

$$
\sum \mu\left(Z_{n}\right) / \nu\left(Z_{n}\right)^{\frac{p}{q}} \geqq C \sum \nu\left(Z_{n}\right)^{1-\frac{p}{q}}=\infty .
$$

However from our assumption (3.2), this is a contradiction.

Thus we have that $\Omega$ is a union of an at most countable number of $\mu$-atoms $Y_{n}$. Let $a_{n}=\mu\left(Y_{n}\right), b_{n}=\nu\left(Y_{n}\right)$. Then we shall show that a Banach space $L^{p}(\mu)$ is linearly isometric to a Banach space $l^{p}\left(a_{n}\right)$. For if $f \in L^{p}(\mu)$, then $f(\omega)$ is constant on $Y_{n}$ for $\mu$-a. e. $\omega$ since $Y_{n}$ is a $\mu$-atom. Hence we may put

$$
f(\omega)=\xi_{n} \quad \text { for } \omega \text { in } Y_{n} .
$$

Putting $\xi=\left(\xi_{n}\right)$, we have that $\xi \in l^{p}\left(a_{n}\right)$. Hence we obtain a linear isometry $V$ of $L^{p}(\mu)$ onto $l^{p}\left(a_{n}\right)$ defined by $V(f)=\xi$.

Then it is easy to show that the image of $F$ by $V$ coincide with $l^{q}\left(b_{n}\right)$.

Since from our assumption (3.2), we have

$$
\sum a_{n} / b_{n}^{\frac{p}{q}}<\infty
$$

Hence it follows from Lemma 2.2 that there exists a finite Borel measure $P$ on $l^{p}\left(a_{n}\right)$ which is quasi-invariant with respect to $l^{q}\left(b_{n}\right)$. Then, $V^{-1} P$ is a finite Borel measure on $L^{p}(\mu)$ which is quasi-invariant with respect to $F$, where the measure $V^{-1} P$ is defined by

$$
V^{-1} P(A)=P(V(A)) \quad \text { for any Borel set } A \text { of } L^{p}(\mu) .
$$

Thus the proof is completed.

REMARK 3.1. (1) In the above theorem, if $1 \leqq q<\infty$, the implication $(1) \Rightarrow(2) \Rightarrow(3) \Rightarrow(4) \Rightarrow(5)$ also hold. However, if $2<q<\infty$, the implication (5) $\Rightarrow$ (1) does not hold (cf. [4, Proposition 4.1.2]).

(2) As was shown in this proof, if one of the five statements of the above theorem be satisfied, then the inclusion $J\left(L^{q}(\nu)\right) \subset L^{p}(\mu)$ holds, so that the Banach space $F$ coincide with $J\left(L^{p}(\nu)\right)$.

Corollary 3.1. Let $1 \leqq p<\infty, 1 \leqq q<\infty$. Suppose that two measures $\mu$ and $\nu$ are mutually equivalent, and that the non-atomic part of $\mu$ has a positive measure. Then there exists no finite Borel measure on $L^{p}(\mu)$ which is quasi-invariant with respect to $L^{q}(\nu) \cap L^{p}(\mu)$. 
ExAmple 3.1. Let $1 \leqq p<\infty, 1 \leqq q<\infty$. Let $\mu$ be a Lebesgue measure on a finite dimensional space $R^{n}$, and let $\nu$ be a $\sigma$-finite Borel measure on $R^{n}$ which is equivalent to $\mu$. Then there exists no finite Borel measure on $L^{p}(\mu)$ which is quasi-invariant with respect to $L^{q}(\nu) \cap L^{p}(\mu)$.

Finally, we shall discuss for an abstract Wiener space (cf. [1]).

Let $1 \leqq p<\infty$. Suppose that two $\sigma$-finite measures $\mu$ and $\nu$ on $(\Omega, \Sigma)$ are mutually equivalent, and also suppose that $L^{2}(\nu)$ is a separable Hilbert space and $L^{2}(\nu) \subset L^{p}(\mu)$. For simplicity, we shall write $H$ instead of $L^{2}(\nu)$. Let $B$ denote the closure of $H$ in $L^{p}(\mu)$, and denote by $i$ the inclusion mapping of $H$ into $B$. Then $B$ forms a separable Banach space with respect to the induced norm by $L^{p}(\mu)$, and it follows from the closed graph theorem that the mapping $i$ of $H$ into $B$ is continuous.

Now as a corollary to Theorem 3.1, we have the following:

COROLlaRY 3.2. The following two statements are equivalent.

(1) The triple $(i, H, B)$ is an abstract Wiener space.

(2) The adjoint mapping $i^{*}$ of $B^{*}$ into $H^{*}$ is absolutely summing.

From this, we have the following:

COROllary 3.3. Under the hypotheses of Corollary 3.2, if ree assume that the non-atomic part of $\mu$ has a positive measure, then the triple $(i, H, B)$ is not an abstract Wiener space.

\section{Acknowledgement}

The author wishes to thank Professor H. Shimomura for his useful comments. 


\section{References}

[1] H. H. KUO: Gaussian Measures in Banach Spaces, Lecture Notes in Math., No. 463, Springer, 1975.

[2] A. Pietsch : Absolut p-summierende Abbildungen in normierten Räumen, Studia Math. 28 (1967), 333-353.

[ 3 ] A. V. SKOROHOD: Integration in Hilbert space, Springer-Verlag Berlin-Heidelberg $\cdot$ New York, 1974.

[4] Y. TAKAHASHI: Quasi-invariant measures on linear topological spaces, Hokkaido Math. J., 4 (1975), 59-81.

[5] Y. TAKAHASHI: On measurable norms and abstract Wiener spaces, Hokkaido Math. J., 6 (1977), 276-283.

[6] Y. TAKAhASHi : Partially admissible shifts on linear topological spaces, Hokkaido Math. J., 8 (1979), 150-166.

[7] D.-X. XIA: Measure and integration theory on infinite dimensional spaces, Academic Press, New York and London, 1972.

Department of Mathematics

Yamaguchi University 